\title{
Blocking Central Opiate Function Modulates Hedonic Impact and Anterior Cingulate Response to Rewards and Losses
}

\author{
Predrag Petrovic, ${ }^{1,2}$ Burkhard Pleger, ${ }^{1}$ Ben Seymour, ${ }^{1}$ Stefan Klöppel, ${ }^{1}$ Benedetto De Martino, ${ }^{1}$ Hugo Critchley, ${ }^{1}$ and \\ Raymond J. Dolan ${ }^{1}$ \\ ${ }^{1}$ Wellcome Trust Centre for Neuroimaging, University College London, London WC1N 3BG, United Kingdom, and ${ }^{2}$ Department of Clinical Neuroscience, \\ Karolinska Institute, 17176 Stockholm, Sweden
}

\begin{abstract}
Reward processing is linked to specific neuromodulatory systems with a dopaminergic contribution to reward learning and motivational drive being well established. Neuromodulatory influences on hedonic responses to actual receipt of reward, or punishment, referred to as experienced utility are less well characterized, although a link to the endogenous opioid system is suggested. Here, in a combined functional magnetic resonance imaging-psychopharmacological investigation, we used naloxone to block central opioid function while subjects performed a gambling task associated with rewards and losses of different magnitudes, in which the mean expected value was always zero. A graded influence of naloxone on reward outcome was evident in an attenuation of pleasure ratings for larger reward outcomes, an effect mirrored in attenuation of brain activity to increasing reward magnitude in rostral anterior cingulate cortex. A more striking effect was seen for losses such that under naloxone all levels of negative outcome were rated as more unpleasant. This hedonic effect was associated with enhanced activity in anterior insula and caudal anterior cingulate cortex, areas implicated in aversive processing. Our data indicate that a central opioid system contributes to both reward and loss processing in humans and directly modulates the hedonic experience of outcomes.
\end{abstract}

Key words: naloxone; opioid; reward; fMRI; cingulate; insula; human

\section{Introduction}

The brains' response to rewards and losses, and learning predictions of their occurrence, have been extensively studied in primates and humans (Schultz, 2000; O’Doherty, 2004). However, less is known regarding how subjective hedonic responses to rewards and losses are mediated. Dopamine is strongly implicated in both reward learning and motivational drive (Berridge, 1996; Schultz, 2000). This role for dopamine contrasts with a putative role for endogenous opiates in mediating hedonic responses to rewards (Berridge, 1996; Peciña et al., 2006).

It has been suggested that hedonic responses are referenced relative to a set point, with specific neuromodulatory systems, including an endogenous opioid system, being implicated in control processes (Koob and Le Moal, 1997). Thus, both activation and attenuation of an endogenous opioid control system might be predicted to have specific hedonic consequences. Indeed, there is evidence that activation of an opioid system induces pleasure (Berridge, 1996; Peciña et al., 2006), whereas blockade enhances aversive responses as seen during induced place aversion in rodents (Narayanan et al., 2004; Skoubis et al., 2005) and dysphoria

\footnotetext{
Received June 18, 2008; revised July 12, 2008; accepted July 28, 2008

This work was supported by a Wellcome Trust Programme Grant (R.J.D.).P.P. was supported by Hjärnfonden and the Swedish Research Council.

Correspondence should be addressed to Predrag Petrovic, Cognitive Neurophysiology, MR Research Center, Department of Clinical Neuroscience, N-8, Karolinska Hospital, 17176 Stockholm, Sweden. E-mail: predrag.petrovi@@ki.se.

DOI:10.1523/JNEUROSCI.2807-08.2008

Copyright $\odot 2008$ Society for Neuroscience $\quad$ 0270-6474/08/2810509-08\$15.00/0
}

in humans (Grevert et al., 1983; Martín del Campo et al., 1992). Moreover, prolonged pain engenders opioid activation (Zubieta et al., 2001; Wagner et al., 2007) with ratings of tonic pain being augmented after naloxone treatment (Koppert et al., 2003, 2005). Such contraregulative processes during an aversive event have been viewed as an adaptation of the organism to stressful challenges, which threaten internal homeostasis (Ochsner and Gross, 2005).

Although species-specific affective motor responses, such as licking and lip-smacking, that follow delivery of a reward have been shown to depend on opioid receptors in nucleus accumbens (Berridge, 1996), this does not allow an unambiguous inference regarding how human hedonic experience is mediated. From the perspective of functional neuroanatomy, it is of interest that brain regions implicated in affective processing, such as anterior cingulate and insula, show considerable overlap with regions responsive to opioids (Casey et al., 2000; Wagner et al., 2001; Petrovic et al., 2002) as well as display high opioid receptor expression (Jones et al., 1991; Willoch et al., 1999, 2004). Indeed, these regions are implicated in reward processing (Knutson et al., 2001; O'Doherty et al., 2003), affective aspects of pain (Vogt and Sikes, 2000), and aversive processing (Büchel and Dolan, 2000; Phillips et al., 2003; Singer et al., 2004). The rostral anterior cingulate cortex (rACC) has a known role in regulation of pain (Petrovic and Ingvar, 2002; Petrovic et al., 2002) and emotion (Bush et al., 2000; Petrovic et al., 2005; Etkin et al., 2006) mediated, at least in part, via the endogenous opioid system (Petrovic et al., 2002; Zubieta et al., 2003, 2005; Wager et al., 2007). Thus, 
this cortical network may be involved in opioid-dependent hedonic response for both losses and rewards.

In this study, our interest was in ascertaining how central opiate function influences human hedonic responses to rewards and punishments, in terms of subjective experience and associated neural responses. We manipulated hedonic response within the context of a gambling task (see Fig. $1 A$ ) that elicited monetary rewards or losses of different magnitudes, while subjects were treated with either a naloxone (a competitive nonselective opioid receptor antagonist) or placebo. We reasoned that blocking an endogenous opioid response should attenuate pleasure associated with reward and augment the aversive intensity of losses, effects that would be expressed in simultaneous modulation of neuronal responses within regions mediating hedonic experience.

\section{Materials and Methods}

Subjects and treatment. Fifteen right-handed healthy male subjects went through the whole scanning procedure (mean age, 24 years; ranging from 20 to 36 years), which was approved by the local ethical committee (Department of Neurology and Neurosurgery, University College London, London, UK). No subject was defined as a pathological gambler according to the South Oaks Gambling Screen (Lesieur and Blume, 1987). The study had a randomized crossover double-blinded design. The subjects participated in experiment on two different days and were treated with naloxone one day and placebo on the other day. Only male subjects were included to avoid risk to unknowingly pregnant female subjects. Three medical doctors were included in the execution of the study. Only one doctor was not blinded to the design; his role was to randomize the treatment and mix the drugs. Oral and written information about risks with naloxone was given to the subjects. Also, a general health questionnaire including both mental and physical disorders and a gambling questionnaire was given to the subjects. Any physical or mental disorder (including pathological gambling), or any drug use was an exclusion criterion. On their first visit, subjects went through a clinical interview and an electrocardiogram to assure that no sign of heart disease was present. Before the study, subjects provided written informed consent.

Each subject was treated on two different occasions, at least $1 \mathrm{~d}$ apart. Naloxone or saline was administered intravenously via a syringe into a forearm. On the naloxone treatment day, $10 \mathrm{mg}$ of naloxone (CP Pharmaceutics) was administered in sterile solution of $0.9 \% \mathrm{NaCl}(25 \mathrm{ml})$ and infused slowly for $5 \mathrm{~min}$, while heart rate was continuously monitored. Blood pressure was monitored before and after the infusion. After the drug administration, the subject was directly moved into the scanner for the scanning procedure (the first scanning session started $\sim 15 \mathrm{~min}$ after treatment). Oxygenation level and heart rate were continuously monitored. The same procedure was applied for the saline treatment day when $25 \mathrm{ml}$ of $0.9 \% \mathrm{NaCl}$ without any active drug was administrated. Subjects had to rate adverse effects/physical symptoms of the drug once before and twice after treatment, once before the functional magnetic resonance imaging (fMRI) session, and once after the fMRI session (see supplemental material, available at www.jneurosci.org). Moreover, subjects had to rate their mood on a 17 item mood scale 15 min after treatment (see supplemental material, available at www.jneurosci.org).

Two subjects were excluded from the analysis: one subject was excluded because he showed highly different mood the two different days $(>2.5$ SD away from the group mean day 2$)$, and one subject was excluded because he had no consistency in his ratings (e.g., rewards were rated as highly unpleasant, although he did not confirm this at the postsession interview). One subject experienced nausea during the first scanning session after he was treated with naloxone. He was excluded from additional participation. Nausea is in healthy non-drug-using subjects an uncommon adverse effect from naloxone, but can be observed incidentally after any scanning session because of claustrophobia, or after intravenous procedures.

Experimental procedure. The gambling task was the same on both days. For each day, the subjects were given a $£ 20$ endowment to gamble. They were not given any information about their total wins or losses until the last day had been completed. In the gambling task, subjects had to choose a left or right wheel of fortune pressing a right or a left key before every new gamble. Each gamble had the same expected outcome of $£ 0$, and there was no difference between the gambles (because we were only interested in how different outcomes were rated without any interference from counterfactual processes related to analysis of the distinct options). The win and loss outcomes in each gamble varied from $£ 1$ to $£ 10$, making it possible to measure processes related specifically to the outcome magnitude. Both the left and right gambles, and the reward and loss outcome in each gamble, were always matched for magnitude to minimize comparative cognitive processes (i.e., if the possible rewards were $\mathfrak{E} 1$, then the possible losses were $-\mathfrak{E} 1$ ). Subjects were told that the outcomes of the wheel of fortune were random. In fact, the total outcome in this gambling

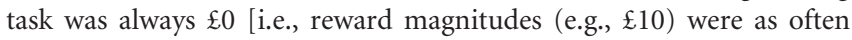
presented as associated loss outcomes (e.g., $-£ 10$ )]. Moreover, we included 20 zero outcomes in each session (i.e., on each day). Thus, there were 60 choices in total that resulted in 20 reward outcomes ( $£ 1$ to $£ 10$ ) (i.e., two of each magnitude), 20 loss outcomes ( $-\mathfrak{E} 1$ to $-\mathfrak{E} 10)$ (i.e., two of each magnitude), and 20 zero outcomes.

After each outcome, subjects completed an affective pleasure rating using a computerized visual analog scale ranging from 100 (strongest imaginable experience of pleasure) to -100 (strongest imaginable experience of unpleasantness), in which zero was defined as neutral affective experience. They had to move a red point on the scale to a position that indicated how they experienced the last outcome using two keys. At the start of rating, the red point appeared randomly on the scale so that the motor planning and movement would not be biased by the outcome. The rating period was $6 \mathrm{~s}$ and was performed after each gamble before the jitter. In the present task, the loss condition induced regret because whenever the chosen gamble lost the other gamble won (Coricelli et al., 2005). However, in the present setting, we were not able to dissociate regret from disappointment and the outcome will contain both effects. We therefore simply defined these events as loss outcomes. There were also 12 null events in which all possible outcomes were 0 that were used to stabilize the baseline (i.e., there were no reward or loss possibilities). The different outcomes were randomized and subjects did not register this manipulation on questioning after the study was completed. The subjects rated their experience of outcomes for each gamble on a visual analog scale from -100 (highest imaginable unpleasantness) to 100 (highest imaginable pleasantness). There was a jittered time of 5-10 $\mathrm{s}$ in between each gamble. The subjects completed two sessions of the gambling task each being $\sim 12$ min long.

Imaging and imaging analysis. The imaging data $\left(\mathrm{T}_{2}{ }^{*}\right.$-weighted echo planar images) measuring blood oxygen level-dependent (BOLD) contrast were acquired using a 1.5 tesla Thermo Fisher Scientific Sonata system. We used a sequence with axial slices tilted by $30^{\circ}$ and a flip angle of $90^{\circ}$ that reduces signal dropout caused by susceptibility-induced field inhomogeneities in amygdala and orbitofrontal cortex (Deichmann et al., 2002). Our field of view covered the whole brain in 44 planes. The repetition time was set to $3.96 \mathrm{~s}$ ( $90 \mathrm{~ms}$ per slice) and echo time to $50 \mathrm{~ms}$ in a single session of $12 \mathrm{~min}$, resulting in 179 volumes.

Images were processed using SPM5 (www.fil.ion.ucl.ac.uk/spm). Scans were realigned, normalized, and spatially smoothed by an $8 \mathrm{~mm}$ full-width half-maximum Gaussian kernel. A high-pass filter (with a cutoff at $128 \mathrm{~s}$ ) was applied to the time series. The data were analyzed as 3-s-long mini-blocks (i.e., using a box-car function) starting from $1 \mathrm{~s}$ before the end of the rotation when the outcome had become obvious and continuing $2 \mathrm{~s}$ afterward as done previously in a similar gambling task (Coricelli et al., 2005). Thus, we had three main conditions using the miniblocks: (1) reward outcome block, (2) loss outcome block, and (3) zero outcome block. On the first (i.e., within-subject) level, we distinguished between conditions from both the naloxone and the placebo day for each subject using general linear model (i.e., the model consisted of several sessions with separate session means). In addition, two separate regressors (i.e., one each for reward or losses) for each drug condition (i.e., naloxone and placebo) represented the parametric modulation of the outcome magnitudes (reward outcome magnitude and loss outcome magnitude). The resulting beta estimate maps were taken to a second- 


\section{A. Gamble}

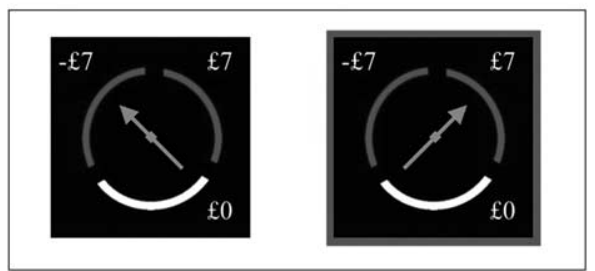

\section{B. Affective Rating of Outcome}

\section{Rating - Losses}

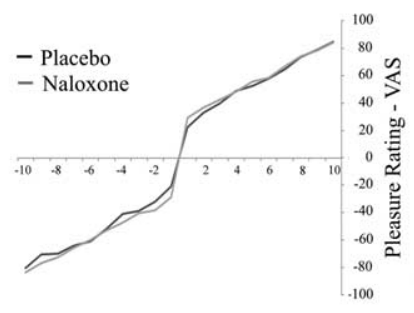

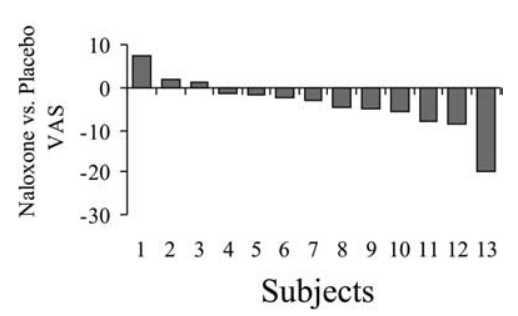

Figure 1. $\quad \boldsymbol{A}$, Gambling task 1. Subjects had to choose a left or right wheel of fortune pressing a right or a left key. Each gamble had the same possible outcomes with an expected value of $£ 0$. The win and loss outcomes in each gamble varied from $£ 1$ to $£ 10$, making it possible to measure responses related to outcome magnitude. Subjects rated how they experienced the outcome of each gamble on a visual analog scale (VAS) from - 100 (highest imaginable unpleasantness) to 100 (highest imaginable pleasantness). $\boldsymbol{B}, \boldsymbol{C}$, Behavioral results. The ratings of the subjects for all outcomes in the placebo and the naloxone day ( $\boldsymbol{B})$. Although treatment did not show any significant difference for overall reward outcomes, a significant effect was observed for the losses in that losses were experienced as more unpleasant after naloxone treatment with 10 of the 13 subjects showing this effect (C).

level group analysis and the significance of contrasts of interest was assessed within a random effects framework (using one-sample $t$ tests) to allow statistical inference across the population.

Our focus of interest in this study was a predefined network known to be involved in positive and negative outcome processing and in opioidrich areas, and included anterior cingulate cortex (ACC), orbitofrontal cortex, insula, amygdala, and ventral striatum (Jones et al., 1991; Willoch et al., 1999; Willoch et al., 2004). We report all activations in these regions as significant where $p<0.001$ (uncorrected). Apart from these regions, we report activations in the rest of the brain only if they reach significance after whole-brain correction.

\section{Results}

\section{Psychological ratings}

Naloxone induced no significant adverse effects (supplemental Table 1, available at www.jneurosci.org as supplemental material) or nonspecific emotional reactions (supplemental Table 2, available at www.jneurosci.org as supplemental material) compared with placebo. Furthermore, we observed no significant difference between naloxone and placebo groups in average pleasure ratings for reward [using visual analog scale ranging from $-100 \mathrm{~mm}$ (i.e., most unpleasant) to $100 \mathrm{~mm}$ (i.e., highest pleasure); placebo: mean, 55.7; SD, 18.42; naloxone: mean, 57.35; SD, 18.19; Wilcoxon's signed ranked test, $p=0.249$ ]. As expected, there was a positive correlation between each outcome $(\mathfrak{E} 1$ to $\mathfrak{E 1 0}$ ) and average pleasure ratings for each reward magnitude ( $£ 1$ to $£ 10$ ) in both the naloxone and the placebo conditions (Spearman's $\rho: r=1.000, p<0.001$ in both conditions) (Fig. $1 B$ ). Strikingly, we found a significant correlation between the treatment differences (placebo vs naloxone) in pleasure ratings for each outcome ( $£ 1$ to $£ 10$ ) and the reward magnitude ( $₹ 1$ to $£ 10$ ) (Spearman's $\rho: r=0.728, p<0.005$ ) (shown for comparison with fMRI findings in Fig. $4 \mathrm{~A}$ ). Thus, under the influence of naloxone, subjects rated reward as less pleasurable when reward magnitude was high (i.e., naloxone attenuated the correlation between pleasure ratings and reward outcome magnitude).
In the loss condition, subjects rated negative outcomes as more aversive after naloxone compared with placebo (pleasantness rating for placebo: mean, -53.22 ; SD, 16.70; naloxone: mean, -56.87 ; SD, 17.88; Wilcoxon's signed ranked test, $p<$ $0.05)$. As expected, there was a positive correlation between each outcome $(-\mathfrak{E} 1$ to $-\mathfrak{E} 10)$ and ratings for each loss magnitude $(-\mathfrak{E} 1$ to $-\mathfrak{E} 10)$ in both the naloxone and placebo conditions (Spearman's $\rho$ : $r=$ $1.000, p<0.001$ in both conditions). When examining for correlation between the treatment induced differences (placebo vs naloxone) in affective rating for loss magnitudes $(-\mathfrak{E} 1$ to $-\mathfrak{E} 10)$ and the loss magnitude ( $-\mathfrak{E} 1$ to $-\mathfrak{E} 10$ ), we found no significant effect $(p=0.321)$. Thus, the results indicates that subjects rated losing more aversive across all levels of losses after naloxone, without a specific impact on high or low losses (shown for comparison with fMRI findings in Fig. 4C).

\section{fMRI}

Reward versus zero outcome blocks

Under placebo, and in accord with previous studies (Knutson et al., 2001), reward outcome blocks (compared with zero outcome blocks) (supplemental Table 3, available at www.jneurosci.org as supplemental material) led to increased BOLD activity in mid- and rostral ACC and basal ganglia, including the ventral striatum (supplemental Fig. $1 A$, available at www.jneurosci.org as supplemental material). In line with our behavioral data we observed no significant difference between the treatments for this contrast [treatment-by-(reward vs zero outcome block) interaction analysis], although there was a trend for greater activation in the midcaudal ACC (mcACC) in the placebo compared with the naloxone condition (supplemental Fig. $1 B$, available at www.jneurosci.org as supplemental material).

\section{Loss versus zero outcome blocks}

Under placebo, loss outcome blocks (compared with zero outcomes blocks) were associated with enhanced activation in rACC, mcACC, posterior cingulate cortex (PCC), bilateral anterior insula, and extrastriate visual cortex (Fig. $2 A$; supplemental Table 4 , available at www.jneurosci.org as supplemental material). A treatment-by-(loss vs zero outcome block) interaction analysis showed enhanced loss related activity under naloxone (compared with placebo) in caudal and subgenual ACC (Fig. $2 B, C$; supplemental Table 4, available at www.jneurosci.org as supplemental material), paralleling our behavioral effect (whereby subjects rated losses as more unpleasant after naloxone treatment). The same contrast also highlighted significantly greater activity in bilateral insula, thalamus, and visual cortex. No enhanced activity was observed in the reverse interaction (placebo vs naloxone).

To assess which brain regions were specifically tied to change in loss affective ratings, we correlated naloxone-induced change in brain activation for loss versus zero outcome blocks (as described in previous paragraph) with naloxone-induced change in affective ratings. This analysis revealed a significant positive correlation between individual naloxone-induced change in affective responses to losses and naloxone-induced change of 


\section{A. Lose vs. Zero Block - Placebo}
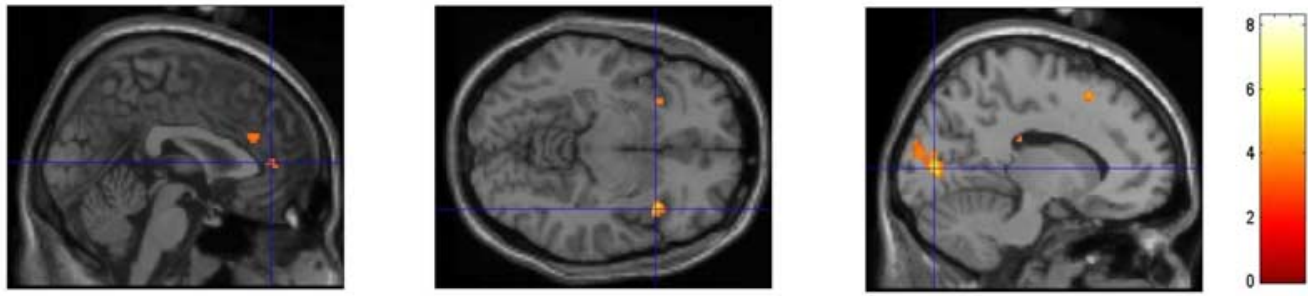

\section{B. Lose vs Zero Block - Naloxone}
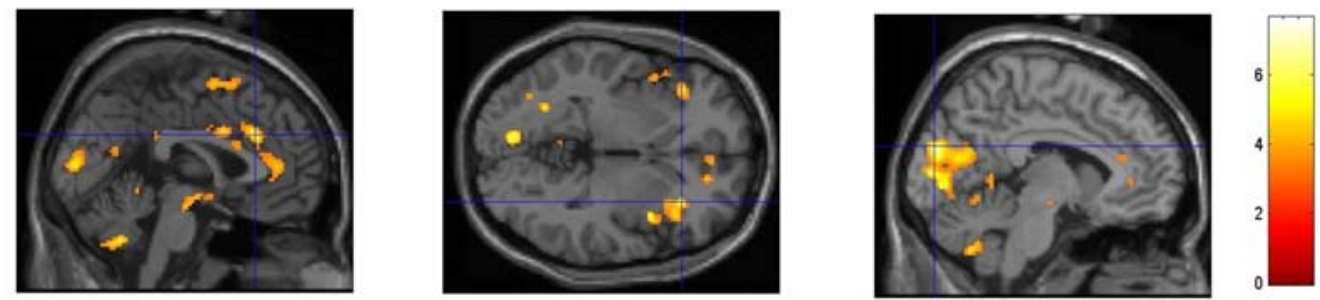

\section{Lose vs. Zero Block - Naloxone vs. Placebo}
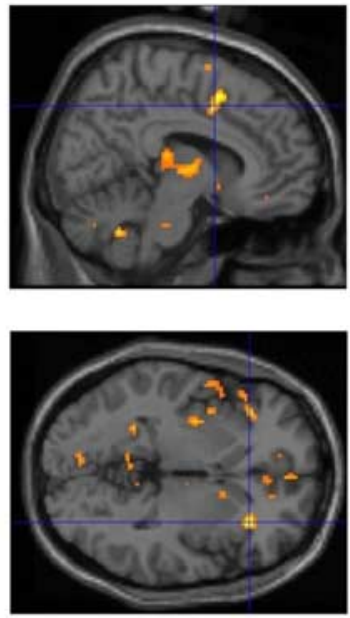
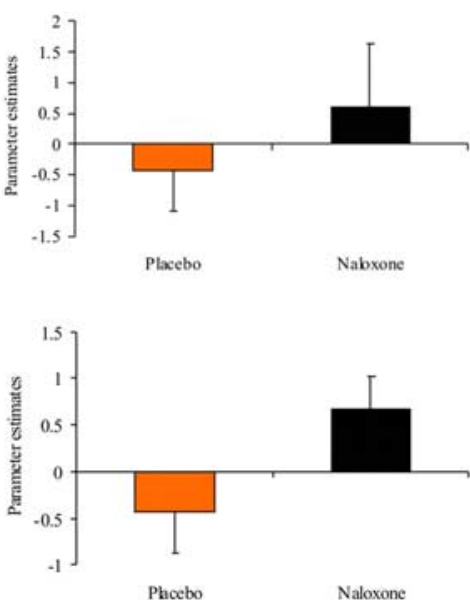
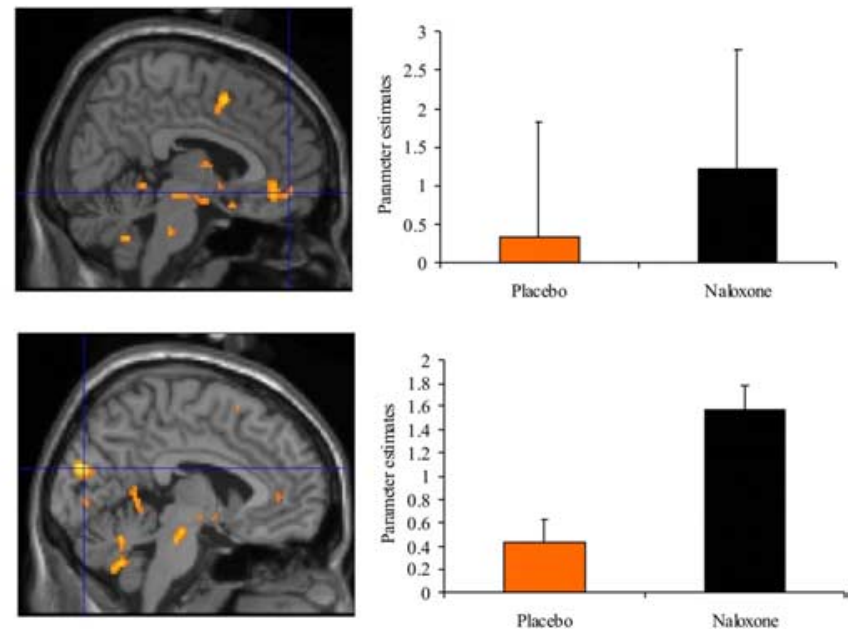

Figure 2. Loss processing under placebo and naloxone. $\boldsymbol{A}$, For placebo, loss versus zero outcome block showed increased activity in midcaudal and rostral ACC, bilateral insula, and visual cortex/precuneus. $\boldsymbol{B}$, The same areas were activated in the naloxone condition. $\boldsymbol{C}$, Testing for an interaction (including the parameter estimates) indicated greater activation for loss versus zero outcome blocks in caudal ACC, bilateral insula, subgenual ACC, precuneus, and extrastriate visual cortex under naloxone compared with placebo treatment. Error bars indicate SEM.

brain activity in caudal ACC (cACC) $([x y z]=4234-12 ; Z=$ 3.89; uncorrected $p>0.001$ ) (supplemental Fig. 2, available at www.jneurosci.org as supplemental material). Thus, within this region, a positive relationship emerged between lossinduced unpleasantness and loss-evoked activity after naloxone treatment.

Regions correlating with outcome magnitude

Next, we assessed whether particular brain regions correlate with outcome magnitude independent of whether these were reward or loss outcomes. Under placebo, activity in rACC and PCC correlated with outcome magnitude independent of value (Fig. $3 A$; supplemental Table 6, available at www.jneurosci.org as supplemental material). Strikingly, these activations were specifically attenuated by naloxone (Fig. $3 B, C$; supplemental Table 6, available at www.jneurosci.org as supplemental material). We also performed separate analysis of reward and loss conditions that showed that rACC correlated with the outcome magnitude inde-

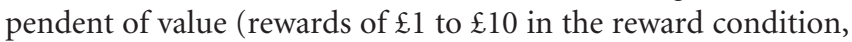
and losses of $\mathfrak{E} 1$ to $\mathfrak{E} 10$ in the loss condition) (supplemental Tables 7, 8, available at www.jneurosci.org as supplemental material). The involvement of rACC in coding reward magnitude has previously been shown (Knutson et al., 2001; O'Doherty et al., 2003). We extend those findings in demonstrating a coding for loss magnitude. This magnitude effect in rACC was significantly attenuated by naloxone for both reward and loss outcome (supplemental Tables 7, 8, available at www.jneurosci.org as supplemental material; Fig. $4 B, D$ ) indicating that the relationship between outcome magnitude and activity in rACC is dependent on the endogenous opioid system, for both rewards and losses. 


\section{Non-value based outcome tracking}

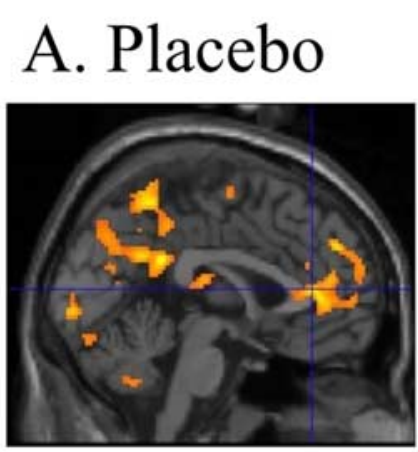

\section{Pl vs. Nal}

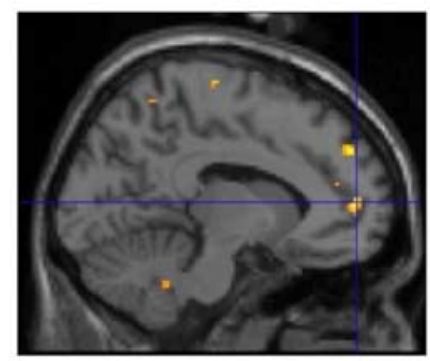

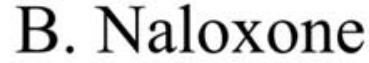
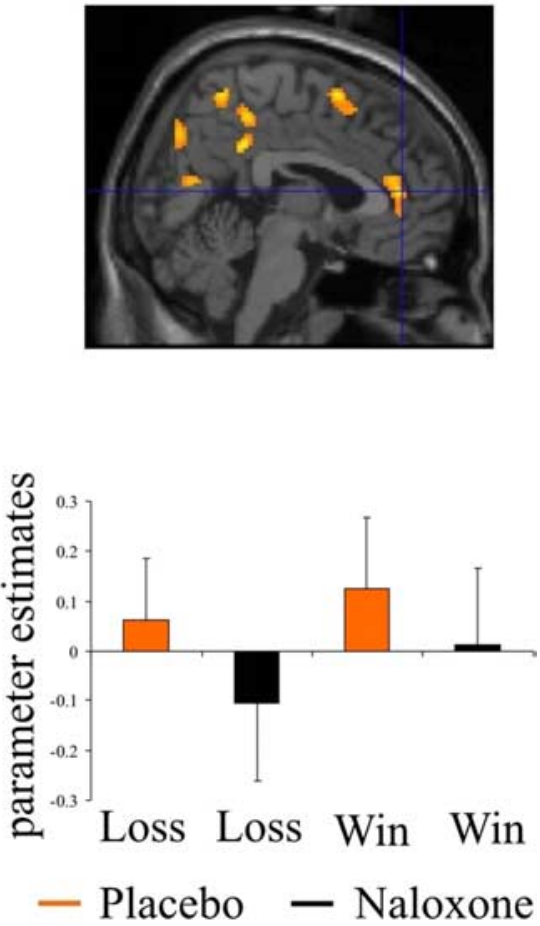

Figure 3. Naloxone effect on outcome magnitude. $\boldsymbol{A}, \boldsymbol{B}$, Activity in $\mathrm{rACC}$ and $\mathrm{PCC}$ correlated with the outcome magnitude independent of value (from $£ 1$ to $£ 10$ for both rewards and losses) for both placebo $(\boldsymbol{A})$ and naloxone $(\boldsymbol{B})$ treatment. $\boldsymbol{C}$, The effect was attenuated in rACC after naloxone treatment and was evident both for losses and rewards. Error bars indicate SEM. PI, Placebo; Nal, naloxone.

\section{Discussion}

Research on how rewards and losses are processed has focused mainly on learning aspects of reward processing, which in the case of reward is closely associated with the dopamine system (Schultz, 2000; O'Doherty, 2004). However, we know little about the mediation of subjective experience of reward and losses, although animal models suggest that the opioid system is involved in reward processing. More specifically, animal research has shown that reward-associated behavior, including motivational behavior, is dependent on the opioid system (Berridge, 1996; Peciña et al., 2006; Baldo and Kelley, 2007). The experience of pleasure after opioid treatment (Berridge, 1996; Peciña et al., 2006) and the involvement of endogenous opioids in pathological gambling (Kim et al., 2001; Grant et al., 2006) also support the idea that this system influences subjective hedonic aspects of rewards.

An opioid involvement in aversive processing has also been suggested. In humans, opioid antagonism attenuates pain ratings primarily when there is expected pain relief (Levine et al., 1978), an effect replicated in subsequent studies (Colloca and Benedetti, 2005). On this basis, it has been suggested that opioid receptor blockade interacts with an expectation pathway (Colloca and Benedetti, 2005) and not on pain processes per se. However, several studies have shown that, if pain is prolonged, opioid antagonists may increase pain ratings even when there is no positive treatment expectation (Koppert et al., 2003, 2005). The opioid system may also attenuate nonpainful aversive responses, as for example induced place aversion in rodents (Narayanan et al., 2004; Skoubis et al., 2005). Different coping strategies are known to modulate emotional responses (Ochsner and Gross, 2005), and there is evidence that an intentional change of emotion can modulate the endogenous opioid system (Zubieta et al., 2003). One possibility is that coping systems invoke the opioid system to dampen aversive responses as seen in the present study. An alternative possibility is that expectation of reward (as in placebo analgesia) induces opioid activation that has effects for both rewards and loss outcomes because they cooccur close in time.

Here, we used naloxone, a competitive opioid receptor antagonist considered to have a slighter higher affinity for $\mu$-receptors than $\delta$ - or $\kappa$-receptors (Corbett et al., 2006) to probe whether the opioid system is involved in the hedonic responses to outcomes of gambles. In line with the aforementioned studies, the data we report indicate that an endogenous opioid system in humans modulates hedonic responses for both losses and rewards. Note that, in the present study, we endeavored to minimize the effects of learning on choice behavior by asking subjects to choose between two identical wheels of fortune in which the expected value always was set to zero. Although it is known that naloxone can itself induce a dysphoric state (Grevert et al., 1983; Martín del Campo et al., 1992, 1994), this effect is more reliable when larger doses are used, relative to that used in the present study, and is primarily observed after several hours of exposure. Thus, the present findings in which there was no overall change in mood or occurrence of dysphoria are more in line with a modulation of an endogenous opioid response.

Our design allowed for two different analyses of the fMRI data, focusing both on differences between the three conditions in terms of miniblocks (reward, loss, and zero outcome blocks), but also a parametric analysis focusing on the reward and loss magnitude in each miniblock (reward and loss outcome magnitude). In terms of block effects, enhanced activity was observed in ACC and bilateral insula for loss compared with zero outcome blocks (Fig. 2A). Note that ACC and anterior insula are strongly implicated in aversive processing, being components of a network suggested to mediate emotional responses to pain (Vogt and Sikes, 2000) and empathy for pain (Singer et al., 2004). Activation in these regions was enhanced under naloxone compared with placebo (Fig. $2 B, C$ ) in line with the increased unpleasantness ratings for losses (Fig. 1C). Thus, the effect we observed is equivalent to augmentation of aversive processing to loss after blockade of the endogenous opioid system. Furthermore, in caudal ACC, loss-induced activity correlated with subjective increases in unpleasantness after naloxone treatment (supplemental Fig. 2, available at www.jneurosci.org as supplemental material). Thus, this psychopharmacological manipulation provides strong support that activity in this region is causal in medi- 
ating hedonic aspect of losses. These findings are in line with suggestion for an opioid role in the control of aversive processing (Zubieta et al., 2001, 2003; Koppert et al., 2003, 2005; Narayanan et al., 2004; Skoubis et al., 2005; Wagner et al., 2007).

We observed enhanced activity in ACC and ventral striatum when reward outcome was compared with zero outcome, as previously shown in other studies of reward processing (Knutson et al., 2001; O’Doherty et al., 2003). In line with our behavioral results, we did not find any difference between the placebo and naloxone treatments in brain activation for reward outcome blocks. Thus, our findings do not support previous assertions that the endogenous opioid system is specifically involved in the hedonic aspect of reward (Berridge, 1996; Peciña et al., 2006), but neither do they exclude this possibility. It is possible that the dose of naloxone we used is too small to block the bulk of cortical opioid receptors. In addition, the hedonic response to reward in the present experiment may not have been of sufficient magnitude to be affected. We note context-dependent resetting of the brain response to rewards has previously been reported (Nieuwenhuis et al., 2005). Thus, it is possible that subjects shift their baseline ratings and brain responses to rewards after the different treatments (yielding a decreased sensitivity in the study). A more sensitive method would be to analyze the magnitude-dependent effects.

rACC activity reflected valenceindependent outcome magnitude (Fig. 3A). Similarly, correlations in the ventromedial prefrontal cortex/rACC have previously been shown only in relation to the value-based reward magnitude (Knutson et al., 2001) and subjective reward value (Kable and Glimcher, 2007). Here, we show that both larger rewards and larger losses were associated with enhanced activity in rACC. Strikingly, naloxone attenuated these effects both for losses and rewards (Figs. $3 B, C, 4 B, D$ ). Thus, activation in rACC that coded for outcome magnitude was susceptible to blockade of the endogenous opioid system independent of outcome valence. Other studies have highlighted the involvement of rACC in placebo analgesia (Petrovic and Ingvar, 2002; Bingel et al., 2006; Kong et al., 2006), an effect known to be partly dependent on the endogenous opioid system (Levine et al., 1978; Colloca and Benedetti, 2005). Opioid activation in rACC has been observed with placebo analgesia as assessed by receptor imaging in human subjects (Zubieta et al., 2005; Wager et al., 2007). rACC has also a role in both emotional attention (Bush et al., 2000; Etkin et al., 2006; Mohanty et al., 2007) and emotional regulation (Etkin et al., 2006; Mohanty et al., 2007). Similar regions to those we identify are implicated in extinction (Phelps et al., 2004; Kalisch et al., 2006) in which an opioid contribution also has been highlighted (Merluzzi et al., 1991; Arntz et al., 1993; Kozak et al., 2007). Thus, there is substantial evidence suggesting this region mediates emotional regulation via an endogenous opioid system. We suggest that the outcome magnitude-related activation in rACC may be

\section{B. Reward magnitude activation} (Pl vs. Nal) (Pl vs. Nal)

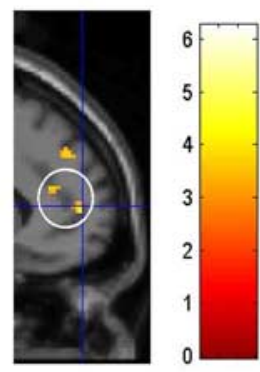

Outcome

\section{Loss magnitude activation}

(Pl vs. Nal)

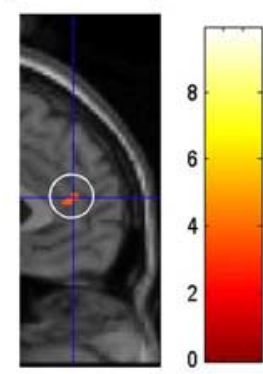

(Pl vs. Nal)
Figure 4. Naloxone effect on outcome magnitude for pleasure ratings and rACC activity in rewards and losses. $A, B$, Naloxone had a more negative effect the larger the reward outcome magnitude was both for affective ratings and activity in $\mathrm{ACCC} . A$, For the havioral ratings, this was manifest in a positive correlation between the treatment difference [placebo (PI) vs naloxone (Nal)] in (Spearman's $\rho: r=0.728 ; p<0.005)$. Thus, large outcome magnitudes were experienced as less pleasurable under naloxone compared with placebo. This effect was mirrored by a Dard outcome magnitude-related activity in $\mathrm{rACC}$ after placebo treatment that was attenuated after naloxone treatment $(\boldsymbol{B})$. $\boldsymbol{C}$ was observed in the $\mathrm{ACC}(\boldsymbol{D})$. Here, the outcome magnitude of loss was attenuated after naloxone treatment. Thus, the correlation between activity in rACC and loss outcome magnitude $(£ 1-£ 10)$ was suppressed by naloxone. Note that the observed magnitude effect in rACC was independent of positive or negative value.

associated with opioid regulation of the hedonic experience in rewards (Berridge, 1996; Peciña et al., 2006) and countering the aversive experience of losses (Ochsner and Gross, 2005). No effects of magnitude were observed in the ventral striatum in line with previous studies showing that the striatal response for rewards are context dependent and adjust adaptively for each new gamble (Nieuwenhuis et al., 2005).

Compared with rostral cingulate, a more caudal region of the cingulate showed an opposite activity profile for losses in the magnitude outcome analysis (i.e., the cACC was more positively correlated with loss magnitude after naloxone treatment). A rostral-caudal division of the ACC has previously been observed in pain and emotion processing [e.g., pain processing is more related with $\mathrm{CACC}$, whereas pain regulation is more related with rACC (Petrovic and Ingvar, 2002; Petrovic et al., 2002)]. Moreover, whereas bottom-up induced pain activates cACC, topdown induced empathy for pain activates more rACC (Singer et al., 2004). Finally, it is specifically the midcaudal part of ACC that is associated with the unpleasantness experience of pain (Rainville et al., 1999). These studies support the idea that cACC is more involved in the hedonic aspect of loss processing, whereas the rACC is more linked to top-down regulation.

Animal research suggests that the opioid-dependent hedonic motor response is processed by ventral striatum, including nucleus accumbens (Berridge, 1996). The endogenous opioid system in ventral striatum may also be involved in mediating some 
motivational behaviors, because opioid injection in nucleus accumbens induces increased intake of palatable food (Baldo and Kelley, 2007). In this regard, it is interesting that we observed no naloxone-induced effect in ventral striatum. There are several reasons for this apparent lack of effect. In the present study, there was no means to increase the consumption of a reward or high degree of hedonic motor responses (such as consistent smiles after reward outcomes) that would relate to striatal-opioid involvement. Instead, the focus was on hedonic experience. We note that the present results are in accord with a recent opioidtracer study showing that a subjective hedonic response, the euphoria in "runner's high," correlates with endogenous opioid increases in rACC and insula, but not within ventral striatum (Boecker et al., 2008). We also note several lines of research that suggest insula involvement in a metarepresentation of the state of the body associated with emotional awareness (Craig, 2002; Critchley et al., 2004) and cingulate cortex involvement in evaluative affective states (Rainville et al., 1999; Vogt and Sikes, 2000; Kable and Glimcher, 2007). On this basis, we argue that our results converge with others to suggest that a cortical system involving the insula and the ACC, in which we observed naloxoneinduced changes in reward and loss processing, relate to hedonic experience.

Finally, naloxone induced a modulatory effect in the outcome magnitude analysis but not in the main effect analysis of reward. It has been suggested that the ventral striatum readjusts its baseline level between each gamble (Nieuwenhuis et al., 2005). In line with that study, we did not observe any correlation with magnitude outcome in ventral striatum either for placebo or naloxone (although such striatal effects were strong in the block analysis). Thus, for each possible reward outcome $(\mathfrak{E} 1-\mathfrak{E} 10)$ at the beginning of the gamble, the striatum responded to a similar degree if there was a subsequent win no matter whether it was $\mathfrak{E} 1$ or $\mathfrak{E} 10$. Therefore, there is good reason to believe that this type of analysis may be insensitive to naloxone effects on reward processing in the ventral striatum.

In conclusion, we show that blocking the opioid system in human healthy subjects modulates hedonic responses for both reward outcome (dependent on magnitude) and loss outcome (independent of the magnitude), and underlying the brain activation. Therefore, the effect of opioid receptor blockade on pathological gambling (Kim et al., 2001; Grant et al., 2006) may be associated with both a decrease in reward experience when winning, but also with an increase in unpleasantness after losing. In terms of decision making, our study speaks to an expression of utility that Bentham referred to as "sovereign masters," reflecting pleasure (reward) and pain (Bentham, 1907). Kahneman has commented that Bentham's concept may reflect a type of "experienced utility" that he distinguishes from "decision utility" that is at the core of canonical utility theory (Kahneman et al., 1997). Although dopamine has been strongly implicated in the latter kind of utility (i.e., decision utility) (Pessiglione et al., 2006), our current findings suggest an opiate contribution to experienced utility.

\section{References}

Arntz A, Merckelbach H, de Jong P (1993) Opioid antagonist affects behavioral effects of exposure in vivo. J Consult Clin Psychol 61:865-870.

Baldo BA, Kelley AE (2007) Discrete neurochemical coding of distinguishable motivational processes: insights from nucleus accumbens control of feeding. Psychopharmacology (Berl) 191:439-459.

Bentham J (1907) An introduction to the principles of morals and legislation. Oxford: Clarendon.
Berridge KC (1996) Food reward: brain substrates of wanting and liking. Neurosci Biobehav Rev 20:1-25.

Bingel U, Lorenz J, Schoell E, Weiller C, Büchel C (2006) Mechanisms of placebo analgesia: rACC recruitment of a subcortical antinociceptive network. Pain 120:8-15.

Boecker H, Sprenger T, Spilker ME, Henriksen G, Koppenhoefer M, Wagner KJ, Valet M, Berthele A, Tolle TR (2008) The runner's high: opioidergic mechanisms in the human brain. Cereb Cortex, in press.

Büchel C, Dolan RJ (2000) Classical fear conditioning in functional neuroimaging. Curr Opin Neurobiol 10:219-223.

Bush G, Luu P, Posner MI (2000) Cognitive and emotional influences in anterior cingulate cortex. Trends Cogn Sci 4:215-222.

Casey KL, Svensson P, Morrow TJ, Raz J, Jone C, Minoshima S (2000) Selective opiate modulation of nociceptive processing in the human brain. J Neurophysiol 84:525-533.

Colloca L, Benedetti F (2005) Placebos and painkillers: is mind as real as matter? Nat Rev Neurosci 6:545-552.

Corbett AD, Henderson G, McKnight AT, Paterson SJ (2006) 75 years of opioid research: the exciting but vain quest for the Holy Grail. Br J Pharmacol 147 [Suppl 1]:S153-S162.

Coricelli G, Critchley HD, Joffily M, O’Doherty JP, Sirigu A, Dolan RJ (2005) Regret and its avoidance: a neuroimaging study of choice behavior. Nat Neurosci 8:1255-1262.

Craig AD (2002) How do you feel? Interoception: the sense of the physiological condition of the body. Nat Rev Neurosci 3:655-666.

Critchley HD, Wiens S, Rotshtein P, Ohman A, Dolan RJ (2004) Neural systems supporting interoceptive awareness. Nat Neurosci 7:189-195.

Deichmann R, Josephs O, Hutton C, Corfield DR, Turner R (2002) Compensation of susceptibility-induced BOLD sensitivity losses in echoplanar fMRI imaging. Neuroimage 15:120-135.

Etkin A, Egner T, Peraza DM, Kandel ER, Hirsch J (2006) Resolving emotional conflict: a role for the rostral anterior cingulate cortex in modulating activity in the amygdala. Neuron 51:871-882.

Grant JE, Potenza MN, Hollander E, Cunningham-Williams R, Nurminen T, Smits G, Kallio A (2006) Multicenter investigation of the opioid antagonist nalmefene in the treatment of pathological gambling. Am J Psychiatry 163:303-312.

Grevert P, Albert LH, Inturrisi CE, Goldstein A (1983) Effects of eight-hour naloxone infusions on human subjects. Biol Psychiatry 18:1375-1392.

Jones AK, Qi LY, Fujirawa T, Luthra SK, Ashburner J, Bloomfield P, Cunningham VJ, Itoh M, Fukuda H, Jones T (1991) In vivo distribution of opioid receptors in man in relation to the cortical projections of the medial and lateral pain systems measured with positron emission tomography. Neurosci Lett 126:25-28.

Kable JW, Glimcher PW (2007) The neural correlates of subjective value during intertemporal choice. Nat Neurosci 10:1625-1633.

Kahneman D, Wakker P, Sarin R (1997) Explorations of experienced utility. Boston: MIT.

Kalisch R, Korenfeld E, Stephan KE, Weiskopf N, Seymour B, Dolan RJ (2006) Context-dependent human extinction memory is mediated by a ventromedial prefrontal and hippocampal network. J Neurosci 26:9503-9511.

Kim SW, Grant JE, Adson DE, Shin YC (2001) Double-blind naltrexone and placebo comparison study in the treatment of pathological gambling. Biol Psychiatry 49:914-921.

Knutson B, Fong GW, Adams CM, Varner JL, Hommer D (2001) Dissociation of reward anticipation and outcome with event-related fMRI. Neuroreport 12:3683-3687.

Kong J, Gollub RL, Rosman IS, Webb JM, Vangel MG, Kirsch I, Kaptchuk TJ (2006) Brain activity associated with expectancy-enhanced placebo analgesia as measured by functional magnetic resonance imaging. J Neurosci 26:381-388.

Koob GF, Le Moal M (1997) Drug abuse: hedonic homeostatic dysregulation. Science 278:52-58.

Koppert W, Angst M, Alsheimer M, Sittl R, Albrecht S, Schüttler J, Schmelz M (2003) Naloxone provokes similar pain facilitation as observed after short-term infusion of remifentanil in humans. Pain 106:91-99.

Koppert W, Filitz J, Tröster A, Ihmsen H, Angst M, Flor H, Schüttler J, Schmelz M (2005) Activation of naloxone-sensitive and -insensitive inhibitory systems in a human pain model. J Pain 6:757-764.

Kozak AT, Spates CR, McChargue DE, Bailey KC, Schneider KL, Liepman 
MR (2007) Naltrexone renders one-session exposure therapy less effective: a controlled pilot study. J Anxiety Disord 21:142-152.

Lesieur HR, Blume SB (1987) The South Oaks Gambling Screen (SOGS): a new instrument for the identification of pathological gamblers. Am J Psychiatry 144:1184-1188.

Levine JD, Gordon NC, Fields HL (1978) The mechanism of placebo analgesia. Lancet 2:654-657.

Martín del Campo AF, McMurray RG, Besser GM, Grossman A (1992) Effect of 12-hour infusion of naloxone on mood and cognition in normal male volunteers. Biol Psychiatry 32:344-353.

Martín del Campo AF, Dowson JH, Herbert J, Paykel ES (1994) Effects of naloxone on diurnal rhythms in mood and endocrine function: a doseresponse study in man. Psychopharmacology (Berl) 114:583-590.

Merluzzi TV, Taylor CB, Boltwood M, Götestam KG (1991) Opioid antagonist impedes exposure. J Consult Clin Psychol 59:425-430.

Mohanty A, Engels AS, Herrington JD, Heller W, Ho MH, Banich MT, Webb AG, Warren SL, Miller GA (2007) Differential engagement of anterior cingulate cortex subdivisions for cognitive and emotional function. Psychophysiology 44:343-351.

Narayanan S, Lam H, Christian L, Levine MS, Grandy D, Rubinstein M, Maidment NT (2004) Endogenous opioids mediate basal hedonic tone independent of dopamine D-1 or D-2 receptor activation. Neuroscience 124:241-246.

Nieuwenhuis S, Heslenfeld DJ, von Geusau NJ, Mars RB, Holroyd CB, Yeung N (2005) Activity in human reward-sensitive brain areas is strongly context dependent. Neuroimage 25:1302-1309.

Ochsner KN, Gross JJ (2005) The cognitive control of emotion. Trends Cogn Sci 9:242-249.

O’Doherty J, Critchley H, Deichmann R, Dolan RJ (2003) Dissociating valence of outcome from behavioral control in human orbital and ventral prefrontal cortices. J Neurosci 23:7931-7939.

O'Doherty JP (2004) Reward representations and reward-related learning in the human brain: insights from neuroimaging. Curr Opin Neurobiol 14:769-776.

Peciña S, Smith KS, Berridge KC (2006) Hedonic hot spots in the brain. Neuroscientist 12:500-511.

Pessiglione M, Seymour B, Flandin G, Dolan RJ, Frith CD (2006) Dopamine-dependent prediction errors underpin reward-seeking behaviour in humans. Nature 442:1042-1045.

Petrovic P, Ingvar M (2002) Imaging cognitive modulation of pain processing. Pain 95:1-5.

Petrovic P, Kalso E, Petersson KM, Ingvar M (2002) Placebo and opioid analgesia-imaging a shared neuronal network. Science 295:1737-1740.

Petrovic P, Dietrich T, Fransson P, Andersson J, Carlsson K, Ingvar M (2005) Placebo in emotional processing-induced expectations of anxiety relief activate a generalized modulatory network. Neuron 46:957-969.
Phelps EA, Delgado MR, Nearing KI, LeDoux JE (2004) Extinction learning in humans: role of the amygdala and vmPFC. Neuron 43:897-905.

Phillips ML, Drevets WC, Rauch SL, Lane R (2003) Neurobiology of emotion perception I: The neural basis of normal emotion perception. Biol Psychiatry 54:504-514.

Rainville P, Carrier B, Hofbauer RK, Bushnell MC, Duncan GH (1999) Dissociation of sensory and affective dimensions of pain using hypnotic modulation. Pain 82:159-171.

Schultz W (2000) Multiple reward signals in the brain. Nat Rev Neurosci 1:199-207.

Singer T, Seymour B, O’Doherty J, Kaube H, Dolan RJ, Frith CD (2004) Empathy for pain involves the affective but not sensory components of pain. Science 303:1157-1162.

Skoubis PD, Lam HA, Shoblock J, Narayanan S, Maidment NT (2005) Endogenous enkephalins, not endorphins, modulate basal hedonic state in mice. Eur J Neurosci 21:1379-1384.

Vogt BA, Sikes RW (2000) The medial pain system, cingulate cortex, and parallel processing of nociceptive information. Prog Brain Res 122:223-235.

Wager TD, Scott DJ, Zubieta JK (2007) Placebo effects on human $\mu$-opioid activity during pain. Proc Natl Acad Sci U S A 104:11056-11061.

Wagner KJ, Willoch F, Kochs EF, Siessmeier T, Tölle TR, Schwaiger M, Bartenstein P (2001) Dose-dependent regional cerebral blood flow changes during remifentanil infusion in humans: a positron emission tomography study. Anesthesiology 94:732-739.

Wagner KJ, Sprenger T, Kochs EF, Tölle TR, Valet M, Willoch F (2007) Imaging human cerebral pain modulation by dose-dependent opioid analgesia: a positron emission tomography activation study using remifentanil. Anesthesiology 106:548-556.

Willoch F, Tolle TR, Wester HJ, Munz F, Petzold A, Schwaiger M, Conrad B, Bartenstein P (1999) Central pain after pontine infarction is associated with changes in opioid receptor binding: a PET study with 11Cdiprenorphine. AJNR Am J Neuroradiol 20:686-690.

Willoch F, Schindler F, Wester HJ, Empl M, Straube A, Schwaiger M, Conrad B, Tölle TR (2004) Central poststroke pain and reduced opioid receptor binding within pain processing circuitries: a $\left[{ }^{11} \mathrm{C}\right]$ diprenorphine PET study. Pain 108:213-220.

Zubieta JK, Smith YR, Bueller JA, Xu Y, Kilbourn MR, Jewett DM, Meyer CR, Koeppe RA, Stohler CS (2001) Regional mu opioid receptor regulation of sensory and affective dimensions of pain. Science 293:311-315.

Zubieta JK, Ketter TA, Bueller JA, Xu Y, Kilbourn MR, Young EA, Koeppe RA (2003) Regulation of human affective responses by anterior cingulate and limbic mu-opioid neurotransmission. Arch Gen Psychiatry 60:1145-1153.

Zubieta JK, Bueller JA, Jackson LR, Scott DJ, Xu Y, Koeppe RA, Nichols TE, Stohler CS (2005) Placebo effects mediated by endogenous opioid activity on $\mu$-opioid receptors. J Neurosci 25:7754-7762. 